\title{
Lemierre's syndrome complicated by an infected branchial cyst
}

\author{
${ }^{1} \mathrm{AA}$ Lwin, ${ }^{2} \mathrm{~S}$ Lohani \\ ${ }^{1}$ Specialist Registrar in Respiratory Medicine and General Internal Medicine; ${ }^{2}$ Consultant Chest Physician, Darent Valley Hospital, Dartford, UK
}

ABSTRACT We present a case of a young man with Lemierre's syndrome characterised by suppurative thrombophlebitis of the internal jugular vein and multiple septic embolic lesions with cavitations in both lungs resulting from an infected branchial cyst which was previously undiagnosed. Lemierre's syndrome is a rare presentation, especially with pulmonary cavitations, but remains a serious illness with a high mortality rate. Early recognition and prompt treatment have significant impact on prognosis. We believe this to be the first case of Lemierre's syndrome complicated by a previously undiagnosed, infected branchial cyst.

Correspondence to AA Lwin, Darent Valley Hospital, Darenth Wood Road, Dartford DA2 8DA, UK

tel. $+44(0) 1322428100$

e-mail aalwin64@yahoo.com

KEYWORDS Branchial cyst, cavitating lung, internal jugular vein thrombosis, Lemierre's syndrome, septic emboli, thrombophlebitis

DECLARATION OF INTERESTS No conflict of interests declared.

\section{CASE REPORT}

A 2 I-year-old Caucasian man presented to the accident and emergency department with a three-week history of pyrexia with sweating, left-sided pleuritic chest pain, productive cough, nausea and vomiting. He denied any history of haemoptysis. The patient also had significant loss of appetite, with a claimed $12 \mathrm{~kg}$ loss of body weight over three weeks. He had received a one-week course of unspecified antibiotic prescribed by his general practitioner without significant improvement. Four days prior to admission, the patient had noted a gradually increasing tender swelling in the left side of his neck. He denied a history of sore throat. He was a smoker of five to six cigarettes per day since the age of 12 years and had smoked cannabis since the age of 14 years. His medical and family history were unremarkable, with no other relevant medical illness of note.

The patient was emaciated. He was febrile, with a temperature of $38.5^{\circ} \mathrm{C}$, tachycardic and had blood pressure of $95 / 59 \mathrm{mmHg}$. His oxygen saturation was $94 \%$ on air and his respiratory rate 20 breaths per minute. There was a $7 \mathrm{~cm}$ red cystic swelling with mild tenderness in the left side of his neck. There was no palpable systemic lymphadenopathy. Heart sounds were normal and his chest was clear on auscultation. Abdominal examination was unremarkable.

Laboratory studies revealed a marked leucocytosis of $21.8 \times 10^{9} / 1$ (neutrophils $18.5 \times 10^{\circ} / 1$ ), a C-reactive protein of $232 \mathrm{mg} / \mathrm{l}$ and an erythrocyte sedimentation rate of $139 \mathrm{~mm}$ in the first hour. Renal function studies showed hyponatraemia of $124 \mathrm{mmol} / \mathrm{l}$, potassium 4.0 $\mathrm{mmol} / \mathrm{l}$ and creatinine $7 \mathrm{I} \mu \mathrm{mol} / \mathrm{l}$. The patient's liver function tests were normal, with the exception of a low albumin of $24 \mathrm{~g} / \mathrm{l}$. Immunoglobulin levels were within the normal range. The patient declined a human

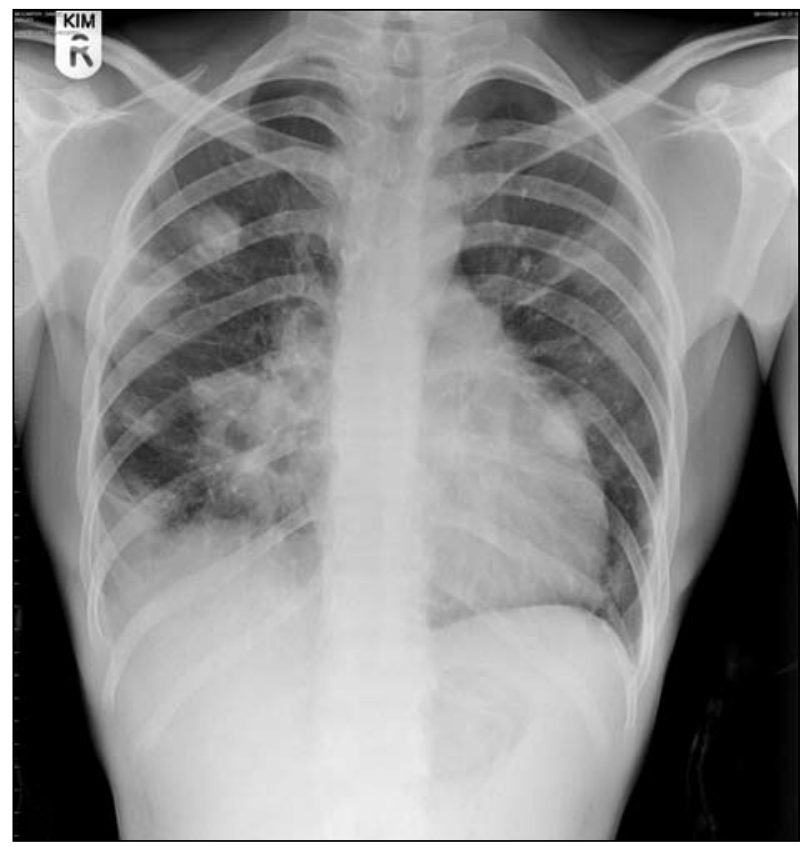

FIGURE I The chest X-ray showed multiple cavitating lesions and opacities.

immunodeficiency virus (HIV) test (the test was offered to him as HIV infection is often seen in young patients in our area and severe sepsis can sometimes be the first presentation, although there was no other suggestive history or laboratory findings).

The patient's chest X-ray (Figure I) revealed multiple cavitating lesions and opacities on both lung fields. An initial differential diagnosis of lung abscesses, pulmonary tuberculosis and lymphoma was made. Intravenous benzylpenicillin and flucloxacillin were commenced while waiting for further microbiological confirmation. A series of sputum cultures grew upper respiratory tract flora only and were negative for acid-fast bacilli. 
A transthoracic echocardiogram did not detect vegetations. A computed tomography scan showed that a necrotic lesion in the neck and multiple pulmonary lesions were probably abscesses originating from the neck (Figures 2 and 3). There was thrombus formation in the left internal jugular vein. Subsequently, intravenous metronidazole was added to the patient's treatment to cover the possibility of anaerobic infection.

The case was reviewed by an ear, nose and throat surgeon. The nose, mouth and oropharynx were normal and the neck swelling was suggested to be compatible with a branchial cyst. Fine needle aspiration revealed an epidermal inclusion cyst with acute and chronic inflammation as well as abundant neutrophils consistent with an abscess. There was no growth of organism from either needle aspirates or blood culture. All these clinical findings fitted with a diagnosis of Lemierre's syndrome originating from an infected branchial cyst.

The clinical condition of the patient and markers of inflammation were significantly improved by intravenous antibiotic treatment. The hyponatraemia resolved. Twenty-four days after admission, the patient was discharged from hospital to complete a total of six weeks' treatment with co-amoxiclav and a three-month course of anticoagulant for internal jugular vein thrombosis. He was well in a follow-up clinic two weeks later and there was a complete resolution of the previously described lesions on a repeat chest X-ray.

\section{DISCUSSION}

Lemierre's syndrome was first described by André Lemierre in 1936' and is also known as necrobacillosis or post-anginal septicaemia. It is now a rare presentation but was a severe septicaemic illness with a high mortality rate $(90 \%)$ in the pre-antibiotic era. Although the prognosis is favourable with early recognition and antibiotics, mortality can still be $4-12 \%$ despite appropriate treatment. ${ }^{2}$

Lemierre's syndrome usually occurs in healthy, immunocompetent young adults, and is characterised most commonly by primary oropharyngeal infection, such as tonsillitis, followed by secondary suppurative thrombophlebitis of the jugular vein and subsequent septic embolisation to other organs. ${ }^{2-4}$

The spread of infection from localised bacterial tonsillitis to Lemierre's syndrome is complex. Several authors believe that the lateral pharyngeal space plays a pivotal role in the spread of infection, as it provides a central connection for other deep neck spaces such as the retropharyngeal and carotid space..$^{5-7}$

In the majority of cases, pus from a tonsillar or peritonsillar abscess spreads deeper into the loose

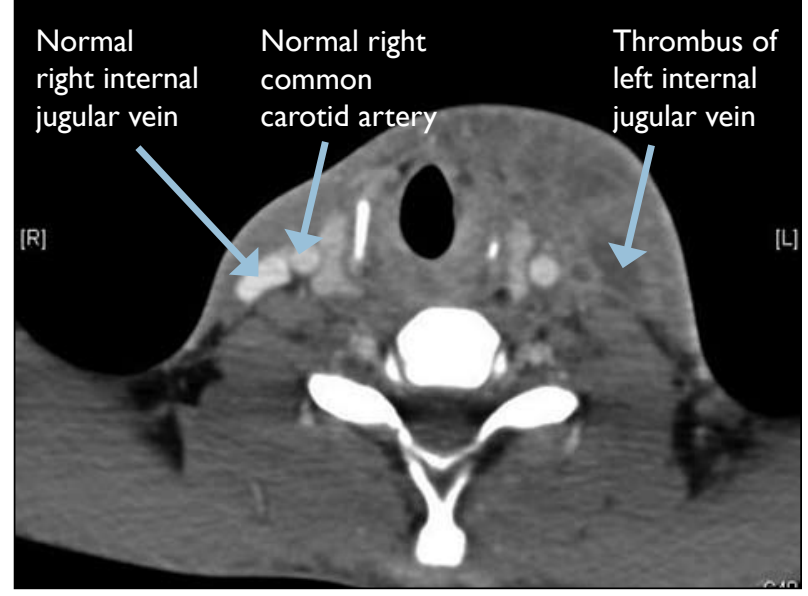

FIGURE 2 Computed tomography scan of the patient's neck, showing necrotic neck swelling in his left side and thrombus in the internal jugular vein.

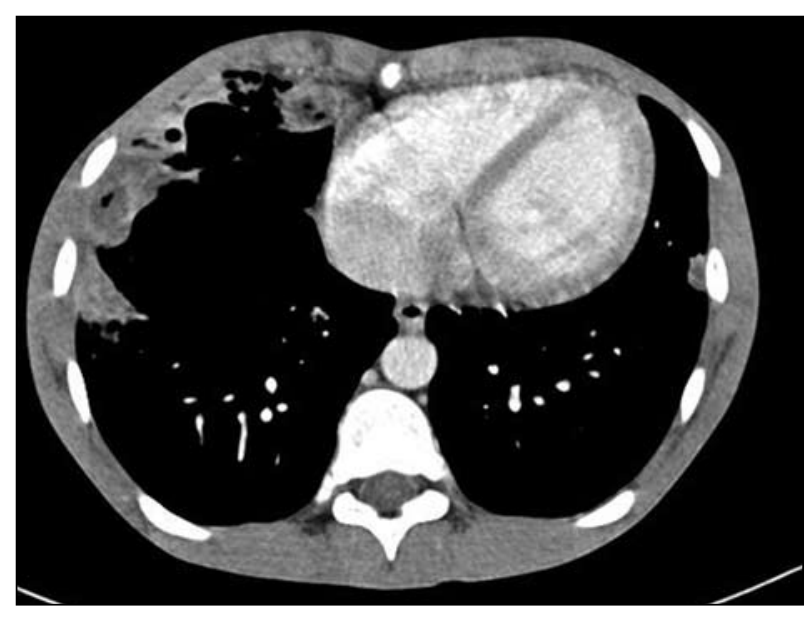

FIGURE 3 Computed tomography of the patient's chest, showing multiple cavitating lesions on both lung fields, mainly on the right side.

pharyngeal space and the wall of the neck veins, causing purulent periphlebitis and endophlebitis. This then spreads to other spaces, such as the retropharygeal space and vessels in the head and neck, and can lead to sinus thrombosis, meningitis or mediastinitis..$^{5-8}$

Some believe that lymphangitis and lymphadenitis in the neck are the primary processes and these then extend into the veins, causing periphlebitis and endophlebitis. However, haematogenous spread via the veins is the most likely route of spread. $5,9,10$

The majority of cases (87.1\%) have pharyngitis or tonsillitis initially; the most frequent site for metastatic infection is the lungs (79.8\%), and $52 \%$ of patients have a tender neck swelling. ${ }^{3}$ In contrast to the pre-antibiotic era, cavitating lesions in lungs are now very rarely seen.

This clinical presentation would include a differential diagnosis of lymphoma or infectious mononucleosis for the neck swelling and staphylococcal infection for the multiple cavitating lesions in the neck and lungs. 
Lymphadenopathy is usually painless and non-tender in lymphoma. Infectious mononucleosis is often considered as it can precede Lemierre's syndrome. The presence of internal jugular vein thrombosis and distant septic metastases are typical features of Lemierre's syndrome. ${ }^{11-13}$

The predominant causative organism in this condition is the anaerobe Fusobacterium necrophorum, a saprophyte of the oropharynx, gastrointestinal tract and genital pathways. Other organisms can also be involved: there are reports of Streptococcus intermedius septicaemia causing Lemierre's syndrome. ${ }^{14}$ There are cases that are culture-negative, ${ }^{15}$ as in our case. This may be because the patient has already been treated with antibiotics prior to admission.

The combination of metronidazole and penicillin/ $\beta$-lactamase inhibitors or carbapenem is recommended to cover the possibility of mixed infection, including streptococci. Metronidazole has excellent activity against all strains of Fusobacterium, good penetration into tissue and excellent oral bioavailability. ${ }^{5,16}$

The pathogenesis of Lemierre's syndrome depends on several factors: virulence of organism; structure and function of tonsillar immune status; concomitant viral infection (such as Epstein-Barr virus); host factors, such as thrombophilia or hypercoagulopathy; and genetic factors, such as single-nucleotide polymorphisms in Tolllike receptors. ${ }^{5}$

One study showed that $F$. necrophorum is tightly clustered in the throat among healthy people in their teens and early 20s, which could explain why Lemierre's syndrome is most common in younger age groups. ${ }^{17}$

Although the oropharynx is the most common primary source of infection, there was no obvious local lesion in the oropharynx in this case. The source of infection was probably from an undiagnosed and pre-existent branchial cyst. We believe this to be the first case report of Lemierre's syndrome caused by an infected branchial cyst.

The role of anticoagulation for jugular vein thrombosis is controversial. Some authors claim that some patients experienced improvement when anticoagulant therapy was added. ${ }^{18}$

\section{CONCLUSION}

Lemierre's syndrome is a rare but potentially fatal disease. An early recognition of the source of infection, prompting an early diagnosis and appropriate antibiotic treatment, is the mainstay of management and improves prognosis.

\section{REFERENCES}

I Lemierre A. On certain septicaemias due to anaerobic organisms. Lancet 1936; I:70I-3. doi:I0.10I6/S0I40-6736(00)57035-4

2 Armstrong AW, Spooner K, Sanders JW. Lemierre's syndrome. Curr Infect Dis Rep 2000; 2:168-73. doi:10.1007/s I 1908-000-0030-z

3 Chirinos JA, Lichtstein DM, Garcia J et al. The evolution of Lemierre syndrome: report of 2 cases and review of the literature. Medicine (Baltimore) 2002; 81:458-65. doi:10.1097/000057922002II000-00006

4 lanniello F, Ferri E, Pinzani A. [Septic thrombophlebitis of the internal jugular vein due to Fusobacterium necrophorum (Lemierre's syndrome). Case report and review of literature.] Acta Otorhinolaryngol Ital 1998; 18:332-7. In Italian.

5 Riordan T. Human infection with Fusobacterium necrophorum (necrobacillosis), with a focus on Lemierre's syndrome. Clin Microbiol Rev 2007; 20:622-59. doi:I 0.I I28/CMR.000 I I-07

6 Blomquist IK, Bayer AS. Life-threatening deep fascial space infections of the head and neck. Infect Dis Clin North Am 1988; 2:237-64.

7 Brook I. Microbiology and management of deep facial infections and Lemierre syndrome. J Otorhinolaryngol Relat Spec 2003; 65: $117-20$.

8 Claus HC. [About 100 cases of septicopyaemia after angina.] Med Klin Wochenschr Praktische Ärzte I93I; 35:I269-7I. In German.

9 Abt IA. Postanginal sepsis. J Pediatr 1932; I:8-15. doi:10.1016/ S0022-3476(32)80039-9

10 Uffenorde W. [The complications of acute laryngitis with particular attention to the involvement of the spatium parapharyngeum.] Leipzig: Curt Kabitzsch; 1925. In German.

II Dagan R, Powell KR. Postanginal sepsis following infectious mononucleosis. Arch Intern Med 1987; I47:I58I-3. doi:10.100I/ archinte.|47.9.158I

12 Møller K, Dreijer B. Post-anginal sepsis (Lemierre's disease): a persistent challenge. Presentation of 4 cases. Scand J Infect Dis 1997; 29:19|-4. doi:I0.3109/00365549709035883

13 Leugers CM, Clover R. Lemierre syndrome: postanginal sepsis. J Am Board Fam Pract 1995; 8:384-9l.

14 Chemlal K, Caby I, Memain $\mathrm{N}$ et al. [Lemierre's syndrome caused by Streptococcus intermedius: an unusual association.] Presse Med 2000; 29:160I-2. In French.

I5 Sherer Y, Mishal J. The changing face of Lemierre's syndrome. Isr Med Assoc J 2003; 5:819-20.

16 Freeman CD, Klutman NE, Lamp KC. Metronidazole. A therapeutic review and update. Drugs 1997; 54:679-708. doi:I0.2165/00003495199754050-00003

17 Jensen A, Hagelskjaer Kristensen L, Prag J. Detection of Fusobacterium necrophorum subsp. funduliforme in tonsillitis in young adults by real-time PCR. Clin Microbiol Infect 2007; 13:69570I. doi:10.1III/j.I469-069I.2007.017I9.x

18 Moore BA, Dekle C,Werkhaven J. Bilateral Lemierre's syndrome: a case report and literature review. Ear Nose Throat J 2002; 81:234-45. 